\title{
Design and Synthesis of Crown Ether Functionalized Imidazoles as a New Type of Building Blocks
}

\author{
SHENG-HUI LI* AND JING XIA
}

\author{
Key Laboratory of Chemical Biology of Hebei Province \\ Key Laboratory of Medicinal Chemistry and Molecular Diagnosis \\ Ministry of Education; College of Chemistry and Environmental Science \\ Hebei University, Baoding 071002, Hebei, P. R. China \\ lish@hbu.edu.cn
}

Received 28 September 2011; Accepted 9 November 2011

\begin{abstract}
A simple, convenient and efficient synthesis for a series of crown ether functionalized imidazoles from benzo-15-crown-5 (B15C5), benzo-18crown-6 (B18C6), imidazole (Im), 2-methylimidazole (mIm), benzimidazole (bIm) and 2-methyl benzimidazole (mbIm) is reported. All these compounds obtained were characterized by IR, ${ }^{1} \mathrm{H}$ NMR, ESI-MS and elemental analysis.
\end{abstract}

Keywords: Imidazole, Benzimidazole, Crown ether, $N$-alkylation, Heterocycles.

\section{Introduction}

The imidazole moiety, as part of the histidine unit in protein, plays a very important role in the biological system. $\mathrm{N}$-Substituted imidazoles, the famous functionalized imidazoles, have attracted considerable attention in recent years, not only due to their valuable pharmacological properties such as antiparasitic, antifungal and antimicrobial activity ${ }^{1-3}$, but also the wide application in supramolecular chemistry ${ }^{4-6}$. Consequently, methodologies for the preparation of functionalized imidazoles have attracted much attention from both industry and academia. Crown ethers have found their widespread use in various fields of science and technology ever since the first preparation of such ligands by Pedersen ${ }^{7}$. During the last two decades the introduction of crown ether function group into various type of molecules is under extensive investigation. These derivatives of crown ethers are attractive compounds for selective metal ion complexing agents ${ }^{8}$, ion selective electrodes preparation ${ }^{9}$ as well as in biological membranes ${ }^{10}$ and pharmaceutical applications ${ }^{11}$.

Up to now, the synthesis of crown ether tethered with imidazole has not been reported. Here we will report a mild, convenient and efficient synthesis for a series of crown ether functionalized imidazoles from benzo-15-crown-5, benzo-18-crown-6, imidazole, 2-methylimidazole, benzimidazole and 2-methyl benzimidazole. The synthetic route is shown in scheme I. These novel crown ether functionalized imidazoles may be used as important intermediates for the synthesis of imidazolium cyclophanes and coordination-driven self-assembly. 


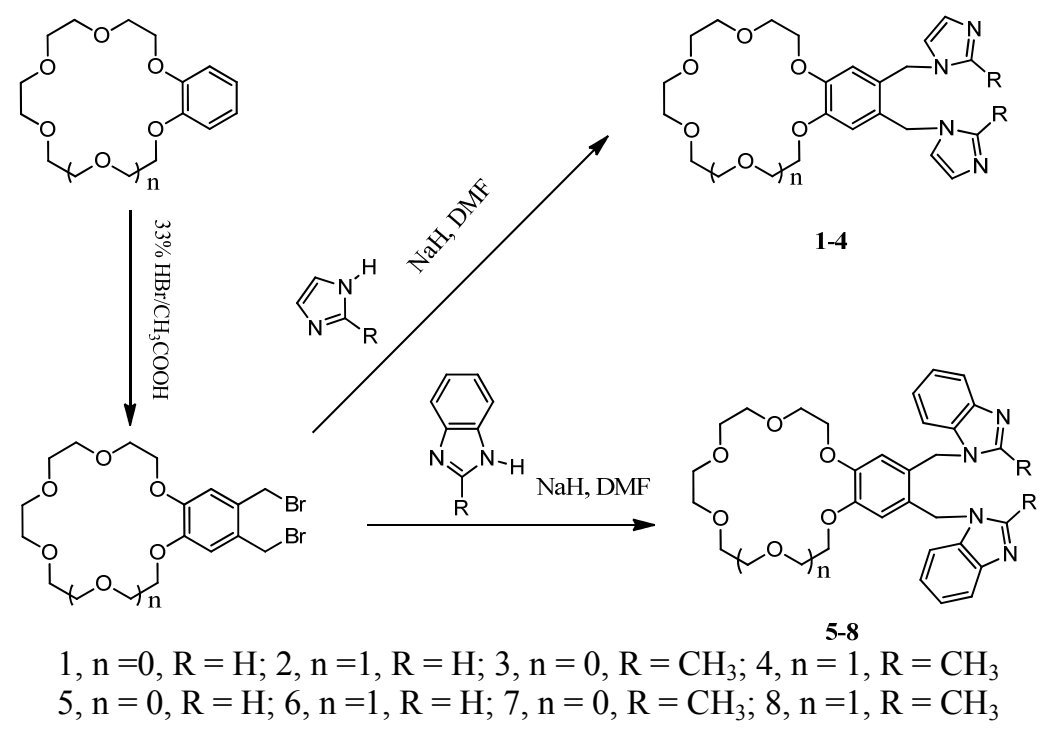

Scheme 1

\section{Experimental}

Melting points were determined on a XT-4 microscopic melting-point spectrometer and are uncorrected. Infrared (IR) spectra were recorded on a BIO-RADFTS-40 analyzer in $\mathrm{KBr} .{ }^{1} \mathrm{H}$ NMR spectra were obtained from solution in $\mathrm{CDCl}_{3}$ with tetramethylsilane (TMS) as internal standard using a Bruker AVANCEIII 600-MHz spectrometer. Elemental analyses were carried out using CE-440 elemental analyzer. The progress of the reaction was monitored by thin-layer chromatography (TLC) using silica gel G (Merck). Starting materials were commercially available unless noted otherwise. $\mathrm{NaH}, 60 \%$ dispension in mineral oil, was washed twice with hexane, dried in vacuo, and stored under Ar. Benzo-15crown-5 and benzo-18-crown-6 were prepared according to the literature procedures ${ }^{7}$. Anhydrous DMF was purified according to the standard method.

\section{Preparation of disubstituted benzo-15-crown-5 and benzo-18-crown-6}

The intermediate compounds, 4',5'-bis(bromomethyl)benzo-15-crown-5 and 4',5'bis(bromomethyl)benzo-18-crown-6 were synthesized in $84 \%$ and $81 \%$ yields respectively, by treating the benzo-15-crown-5 or benzo-18-crown- 6 with $33 \% \mathrm{HBr}$ /acetic acid solution in the presence of paraformaldehyde according to the reported method ${ }^{12}$.

\section{General procedure for preparation of crown ether functionalized imidazoles}

To a solution of imidazole $(68 \mathrm{mg}, 1 \mathrm{mmol})$ in $10 \mathrm{~mL}$ dry DMF under nitrogen, $\mathrm{NaH}$ $\left(67 \mathrm{mg}, 2.75 \mathrm{mmol}\right.$ ) was added at $0^{\circ} \mathrm{C}$. The mixture was stirred at the same temperature for $30 \mathrm{~min}$, then 4',5'-bis(bromomethyl)benzo-15-crown-5 (227 mg, $0.5 \mathrm{mmol})$ in $15 \mathrm{~mL}$ of DMF was added dropwise over a period of $3 \mathrm{~h}$ and the mixture was stirred at room temperature for $20 \mathrm{~h}$. After the reaction was completed, the solvent was removed under reduced pressure and the residue was washed with water and extracted with dichloromethane. After drying over $\mathrm{MgSO}_{4}$, the solution was taken to dryness, the crude product was column chromatographed with dichloromethane/methanol $(8: 1 \mathrm{v} / \mathrm{v})$ as eluant to give the desired product. The related data of crown ether functionalized imidazoles are collected as follows: 


\section{4',5'-Bis((1H-imidazol-1-yl)methyl)benzo-15-crown-5(1)}

Yield 69\%; white solid; m.p. 182-184; ${ }^{1} \mathrm{H}$ NMR $\left(\mathrm{CDCl}_{3}, 600 \mathrm{MHz}\right) \delta: 7.43$ (s, 2H, Im -1-yl-H), 7.10 (s, 2H, Im -1-yl-H ), 6.76 (s, 2H), 6.54 (s, 2H, Ar-H), 4.98 ( s, 4H,-CH $), 4.12$ (t, 4H, $\left.\mathrm{CH}_{2}\right), 3.88$ (t, $\left.4 \mathrm{H},-\mathrm{CH}_{2}\right), 3.65\left(\mathrm{~s}, 8 \mathrm{H},-\mathrm{CH}_{2}\right)$; $\mathrm{IR}(\mathrm{KBr}) v_{\max } / \mathrm{cm}^{-1}: 3737,3421,3096,2928$, 2850, 1658, 1611, 1548, 1362, 1269, 1224, 1128, 1044, 937, 876, 755; m/z(ESI) calcd. for: $\mathrm{C}_{22} \mathrm{H}_{28} \mathrm{~N}_{4} \mathrm{O}_{5} \quad[\mathrm{M}+\mathrm{Na}]^{+} .451 .1$; Elemental analysis: $\mathrm{C}_{22} \mathrm{H}_{28} \mathrm{~N}_{4} \mathrm{O}_{5}$ found: $\mathrm{C}: 61.66, \mathrm{H}: 6.58$, $\mathrm{N}: 13.09$, O:18.67; cal.C:61.48, H:6.68, N:13.18, O:18.66.

\section{4',5'-Bis((1H-imidazol-1-yl)methyl)benzo-18-crown-6(2)}

Yield 77\%; white solid; m.p.126-128; ${ }^{1} \mathrm{H}$ NMR $\left(\mathrm{CDCl}_{3}, 600 \mathrm{MHz}\right) \delta: 7.42(\mathrm{~s}, 2 \mathrm{H},-\mathrm{CH})$, 7.09 (s, 2H, -CH), 6.76 (s, 2H, -CH), 6,59 (s, 2H, Ar-H), $4.94\left(\mathrm{~s}, 4 \mathrm{H},-\mathrm{CH}_{2}\right), 4.08(\mathrm{t}, 4 \mathrm{H},-$ $\left.\mathrm{CH}_{2}\right), 3.89$ (t, 4H, $\left.-\mathrm{CH}_{2}\right), 3.74\left(\mathrm{t}, 4 \mathrm{H},-\mathrm{CH}_{2}\right), 3.70$ (s, $\left.8 \mathrm{H},-\mathrm{CH}_{2}\right)$; IR( $\left.\mathrm{KBr}\right)$ vmax/cm-1: 3743, 3413, 2914, 1611, 1569, 1513, 1453, 1417, 1172, 1050, 833, 738, 594; m/z(ESI) calcd. for: $\mathrm{C}_{24} \mathrm{H}_{34} \mathrm{~N}_{4} \mathrm{O}_{6} \quad[\mathrm{M}+\mathrm{Na}]^{+} .474 .5$; Elemental analysis: $\mathrm{C}_{24} \mathrm{H}_{34} \mathrm{~N}_{4} \mathrm{O}_{6}$ found: C:60.74, $\mathrm{H}: 7.22$, $\mathrm{N}: 11.81, \mathrm{O}: 20.23$; cal.C:60.68, H: 7.08, N:11.87, O:18.87.

4',5'-Bis((2-methyl-1H-imidazol-1-yl)methyl)benzo-15-crown-5(3)

Yield 61\%; white solid; m.p.66-67 ; ${ }^{1} \mathrm{H}$ NMR $\left(\mathrm{CDCl}_{3}, 600 \mathrm{MHz}\right) \delta: 6.96$ (s, 2H, Im -1-yl$\mathrm{H}), 6.65(\mathrm{~s}, 2 \mathrm{H}), 6.32(\mathrm{~s}, 2 \mathrm{H}, \mathrm{Ar}-\mathrm{H}), 4.85\left(\mathrm{~s}, 4 \mathrm{H},-\mathrm{CH}_{2}\right), 3.99\left(\mathrm{t}, 4 \mathrm{H},-\mathrm{CH}_{2}\right), 3.86-3.84(\mathrm{t}, 4 \mathrm{H}$, $\left.\mathrm{CH}_{2}\right), 3.74\left(\mathrm{~s}, 8 \mathrm{H},-\mathrm{CH}_{2}\right), 2.30\left(\mathrm{~s}, 6 \mathrm{H},-\mathrm{CH}_{3}\right)$; IR(KBr): vmax/cm-1 3740, 3106, 2920, 2867, $1658,1609,1518,1362,1274,1210,1129,1040,940,893,735,592 ; \mathrm{m} / \mathrm{z}(\mathrm{ESI})$ calcd. for: $\mathrm{C}_{24} \mathrm{H}_{34} \mathrm{~N}_{4} \mathrm{O}_{5}[\mathrm{M}+\mathrm{Na}]^{+} .471 .2$; Elemental analysis: $\mathrm{C}_{24} \mathrm{H}_{34} \mathrm{~N}_{4} \mathrm{O}_{5}$ found:C:62.86, $\mathrm{H}: 7.47$, $\mathrm{N}: 12.22$, O:17.45; cal.C:62.75, H:7.61, N:12.18, O:18.68.

\section{4',5'-Bis((2-methyl-1H-imidazol-1-yl)methyl)benzo-18-crown-6(4)}

Yield 70\%; white solid; m.p.68-70 ${ }^{\circ} \mathrm{C} ;{ }^{1} \mathrm{H}$ NMR $\left(\mathrm{CDCl}_{3}, 600 \mathrm{MHz}\right) \delta: 6.99$ (s, 2H, -CH), $6.65(\mathrm{~s}, 2 \mathrm{H},-\mathrm{CH}), 6.34$ (s, 2H, $-\mathrm{Ph}-\mathrm{H}), 4.85\left(\mathrm{~s}, 4 \mathrm{H},-\mathrm{CH}_{2}\right), 4.01\left(\mathrm{t}, 4 \mathrm{H},-\mathrm{CH}_{2}\right), 3.86(\mathrm{t}, 4 \mathrm{H},-$ $\left.\mathrm{CH}_{2}\right), 3.74\left(\mathrm{t}, 4 \mathrm{H},-\mathrm{CH}_{2}\right), 3.70\left(\mathrm{~s}, 8 \mathrm{H},-\mathrm{CH}_{2}\right), 2.30\left(\mathrm{~s}, 6 \mathrm{H},-\mathrm{CH}_{3}\right) ; \mathrm{IR}(\mathrm{KBr}) v \mathrm{max} / \mathrm{cm}-1$ : 3737, 2924, 2338, 1609, 1592, 1524, 1452, 1415, 1351, 1270, 1202, 1103, 1049, 954, 752; $\mathrm{m} / \mathrm{z}(\mathrm{ESI})$ calcd. for: $\mathrm{C}_{26} \mathrm{H}_{38} \mathrm{~N}_{4} \mathrm{O}_{6}[\mathrm{M}+\mathrm{Na}]^{+} .523 .3$; Elemental analysis: $\mathrm{C}_{26} \mathrm{H}_{38} \mathrm{~N}_{4} \mathrm{O}_{6}$ found: C:62.13, H:7.62, N:11.15, O: 19.10; cal.C:62.08, H:7.78, N:11.07, O:19.15.

\section{4',5'-Bis((1H-benzo[d]imidazol-1-yl)methyl)benzo-15-crown-5(5)}

Yield 80\%; white solid; m.p. $102-104^{\circ} \mathrm{C} ;{ }^{1} \mathrm{H}$ NMR $\left(\mathrm{CDCl}_{3}, 400 \mathrm{MHz}\right) \delta: 7.82$ (d, J = $7.9 \mathrm{~Hz}$, 2H, Ph-H), 7.73 (s, 2H, -CH), 7.27 (m, 4H, Ph-H), 7.10 (d, J = 7.9 Hz, 2H, Ph-H), 6.59 (s, 2H, Ph-H), 5.17 (s, 4H, Ph-H), 3.96 (t, J =4.3 Hz, 4H, $\left.-\mathrm{CH}_{2}\right), 3.83$ (t, J =4.3 Hz, 4H, $-\mathrm{CH}_{2}$ ), $3.71\left(\mathrm{~s}, 8 \mathrm{H},-\mathrm{CH}_{2}\right)$; IR(KBr) vmax/cm-1: 3740, 3340, 3050, 2917, 2866, 2328, 1655, 1613, $1518,1459,1419,1363,1269,1129,1042,891,852,742,519 ; \mathrm{m} / \mathrm{z}(\mathrm{ESI})$ calcd. for: $\mathrm{C}_{30} \mathrm{H}_{32} \mathrm{~N}_{4} \mathrm{O}_{5}[\mathrm{M}+\mathrm{H}]^{+} .529 .6$; Elemental analysis: $\mathrm{C}_{30} \mathrm{H}_{32} \mathrm{~N}_{4} \mathrm{O}_{5}$ found: C:68.17, $\mathrm{H}: 6.10$, $\mathrm{N}: 10.60, \mathrm{O}: 15.13$; cal.C:68.45, H:6.01, N:10.38, O:15.56.

\section{4',5'-Bis((1H-benzo[d]imidazol-1-yl)methyl)benzo-18-crown-6(6)}

Yield 84\%; white solid; m.p.156-159; ${ }^{1} \mathrm{H}$ NMR $\left(\mathrm{CDCl}_{3}, 600 \mathrm{MHz}\right) \delta: 8.18(\mathrm{~s}, 2 \mathrm{H},-\mathrm{CH})$, 7.82 (d, J =7.9 Hz, 2H, Ph-H), 7.73 (s, 2H, -CH), 7.27 (m, 2H, Ph-H), 7.10 (d, J = 7.9 Hz, 2H, Ph-H), 6.59 (s, 2H, Ph-H), 5.17 (s, 4H, $\left.\mathrm{CH}_{2}\right), 3.96$ (t, J=4.3 Hz, 4H, $\left.-\mathrm{CH}_{2}\right), 3.83$ (t, J=4.3 $\left.\mathrm{Hz}, 4 \mathrm{H},-\mathrm{CH}_{2}\right), 3.71\left(\mathrm{~s}, 8 \mathrm{H},-\mathrm{CH}_{2}\right) ; \mathrm{IR}(\mathrm{KBr}) v \max / \mathrm{cm}-1: 3738,2913,2335,1612,1557$, $1523,1459,1354,1278,1201,1104,1010,893,749 ; \mathrm{m} / \mathrm{z}(\mathrm{ESI})$ calcd. for: $\mathrm{C}_{32} \mathrm{H}_{38} \mathrm{~N}_{4} \mathrm{O}_{6}$ $[\mathrm{M}+\mathrm{Na}]^{+}$. 597.6 Elemental analysis: $\mathrm{C}_{32} \mathrm{H}_{38} \mathrm{~N}_{4} \mathrm{O}_{6}$ found: $\mathrm{C}: 66.88, \mathrm{H}: 6.67, \mathrm{~N}: 9.75, \mathrm{O}$ : 16.70; cal.C: 66.57, H: 6.75, N: 10.04, O: 16.75 . 


\section{4',5'-Bis((2-methyl-1H-benzo[d]imidazol-1-yl)methyl)benzo-15-crown-5(7)}

Yield 78\%; white solid; m.p. $142-143{ }^{\circ} \mathrm{C} ;{ }^{1} \mathrm{H}$ NMR $\left(\mathrm{CDCl}_{3}, 400 \mathrm{MHz}\right) \delta: 7.73$ ( d, J = 7.9 Hz, 2H, Ph-H), 7.24 ( t, J = 7.7 Hz, 2H, Ph-H), 7.17 ( t, J = 7.5 Hz, 2H, Ph-H), 7.02 ( t, J = 7.9 $\mathrm{Hz}, 2 \mathrm{H}, \mathrm{Ph}-\mathrm{H}), 6.15$ (s, 2H, Ph-H), 5.24 (s, 4H,-CH $), 3.71$ (br s, 4H,-CH $), 3.68$ (br s , 4H,$\left.\mathrm{CH}_{2}\right), 3.62\left(\mathrm{~s}, 8 \mathrm{H},-\mathrm{CH}_{2}\right), 2.49\left(\mathrm{~s}, 6 \mathrm{H},-\mathrm{CH}_{3}\right) ; \mathrm{IR}(\mathrm{KBr}) v \max / \mathrm{cm}-1: 3740,3381,2921,2863$, 2364, 1763, 1613, 1513, 1459, 1419, 1363, 1269, 1129, 1049, 938, 745, 740; m/z(ESI) calcd. for: $\mathrm{C}_{32} \mathrm{H}_{36} \mathrm{~N}_{4} \mathrm{O}_{5}[\mathrm{M}+\mathrm{Na}]^{+} .579 .6$; Elemental analysis: $\mathrm{C}_{32} \mathrm{H}_{36} \mathrm{~N}_{4} \mathrm{O}_{5}$ found: $\mathrm{C}: 69.05$, $\mathrm{H}: 6.52$, N:10.06, O:14.37; cal.C:68.85, H:6.71, N:10.18, O:14.56,

\section{4',5'-Bis((2-methyl-1H-benzo[d]imidazol-1-yl)methyl)benzo-18-crown-6(8)}

Yield 79\%; white solid; m.p.142-144 ${ }^{\circ} \mathrm{C} ;{ }^{1} \mathrm{H}$ NMR $\left(\mathrm{CDCl}_{3}, 600 \mathrm{MHz}\right) \delta: 7.82(\mathrm{~d}, \mathrm{~J}=7.9 \mathrm{~Hz}$, 2H, Ph-H), 7. 72 (m, 4H, Ph-H), 7.10 (d, J=7.9 Hz, 2H, Ph-H), 6.59 (s, 2H, Ph-H), 5.17 (s, $\left.4 \mathrm{H},-\mathrm{CH}_{2}\right), 4.22\left(\mathrm{t}, 4 \mathrm{H},-\mathrm{CH}_{2}\right), 3.96\left(\mathrm{t}, \mathrm{J}=4.3 \mathrm{~Hz}, 4 \mathrm{H},-\mathrm{CH}_{2}\right), 3.83\left(\mathrm{t}, \mathrm{J}=4.3 \mathrm{~Hz}, 4 \mathrm{H},-\mathrm{CH}_{2}\right)$, $3.71\left(\mathrm{~s}, 8 \mathrm{H},-\mathrm{CH}_{2}\right), 2.54\left(\mathrm{~s}, 6 \mathrm{H}, \mathrm{CH}_{3}\right)$; IR( $\left.\mathrm{KBr}\right)$ vmax/cm-1: 3738, 3423, 3009, 2905, 2826, $1624,1573,1513,1459,1292,1221,1101,1026,887,753 ; \mathrm{m} / \mathrm{z}(\mathrm{ESI})$ calcd.for: $\mathrm{C}_{34} \mathrm{H}_{42} \mathrm{~N}_{4} \mathrm{O}_{6}$ $[\mathrm{M}+\mathrm{Na}]^{+} .625 .3$; Elemental analysis: $\mathrm{C}_{34} \mathrm{H}_{42} \mathrm{~N}_{4} \mathrm{O}_{6}$ found: C:67.75, H:7.02, N:9.30, O: 15.93; cal.C:67.57, H:7.25, N:9.43, O:15.75.

\section{Results and Discussion}

Imidazoles can be conveniently $N$-alkylated when treated with base followed by an alkyl halide or sulfonate, However, $\mathrm{N}$-alkylation of substituted imidazoles gives regioisomers in most cases. The ratio of isomers is difficult to predict, as the regiochemical outcome generally is dependent on the structure of both imidazole and alkylating agent as well as the exact reaction conditions used ${ }^{13-22}$.

In presence of $\mathrm{NaH}$, imidazole derivatives such as $\mathrm{Im}, \mathrm{mIm}$, bIm and mbIm reacted with 4',5'-bis(bromomethyl)benzo-15-crown-5 or 4',5'-bis(bromomethyl)benzo-18-crown-6 afforded the corresponding crown ether functionalized imidazoles in moderate to good yield. The results are summarized Table 1 .

All final crown ether functionalized imidazoles 1-8 are easily soluble in dichloromethane, chloroform, acetone, ethanol and methanol et al. Their structures were characterized by different spectroscopic technique such as IR, ${ }^{1} \mathrm{H}$ NMR, ESI-MS and elemental analysis. The elemental analyses are in good agreement with compounds 1-8 respectively. The ESI-MS spectra reveal that the molecular ion peaks and fragmentation peaks are in accordance with the given structures of 1-8. In the ${ }^{1} \mathrm{H}$ NMR spectra of the synthesized compounds we observed all proton signals of aromatic rings, heterocyclic rings and methylene groups, and did not observe protons of the $\mathrm{NH}$ group of heterocyclic rings of the initial compounds.

Table 1 summarizes the main results obtained in these $N$-alkylation reactions, from which the reactivity order bIm $>$ mbIm $>\operatorname{Im}>$ mIm can be found. This maybe explained by the steric effect and the weak electron-donating effect of methyl group in the imidzole ring and the conjugative effect of the phenyl ring fused imidzole.

\section{Conclusion}

In conclusion, we have described a convenient, mild and efficient method for a series of crown ether functionalized imidazoles from benzo-15-crown-5, benzo-18-crown-6, imidazole, 2-methylimidazole, benzimidazole and 2-methyl benzimidazole. All these compounds obtained were characterized by IR, ${ }^{1} \mathrm{H}$ NMR, ESI-MS and elemental analysis. The synthesis of crown ether functionalized imidazolium cyclophanes and coordinationdriven self-assembly are currently being explored in our lab. 
Table 1. Synthesis of crown ether functionalized imidazoles.

\begin{tabular}{ccccccc}
\hline Entry & imidzaole & Crown ether & Temp. ${ }^{\circ} \mathrm{C}$ & $\begin{array}{c}\text { Reaction } \\
\text { time, } \mathrm{h}\end{array}$ & m.p. $\left({ }^{\circ} \mathrm{C}\right)$ & $\begin{array}{c}\text { Isolated } \\
\text { Yield, \% }\end{array}$ \\
\hline 1 & $\mathrm{Im}$ & B15C5 & 25 & 24 & $182-184$ & 69 \\
2 & $\mathrm{Im}$ & B18C6 & 25 & 24 & $126-128$ & 77 \\
3 & $\mathrm{mIm}$ & B15C5 & 25 & 24 & $66-67$ & 61 \\
4 & $\mathrm{mIm}$ & B18C6 & 25 & 24 & $68-70$ & 71 \\
5 & $\mathrm{bIm}$ & B15C5 & 25 & 24 & $102-104$ & 80 \\
6 & $\mathrm{bIm}$ & B18C6 & 25 & 24 & $156-159$ & 84 \\
7 & $\mathrm{mbIm}$ & B15C5 & 25 & 24 & $142-143$ & 78 \\
8 & $\mathrm{mbIm}$ & B18C6 & 25 & 24 & $142-144$ & 79 \\
\hline
\end{tabular}

\section{Acknowledgment}

The project was supported by the Pharmaceutical Joint Research Fundation of the Natural Science Foundation of Hebei Province and China Shiyao Pharmaceutical Group Co., Ltd. (B2011201174) and Open Fund of Laboratory of Chemical Biology of Hebei Province (No. 09265631D-7).

\section{References}

1. Mukherjee A, Kumar S, Seth M and Bhaduri A P, Ind J Chem., 1989, 28B, 391.

2. Norman S M, Bennett R D, Poling S M, Maier V P and Nelson M D, Plant Physiol., 1986, 80, 122.

3. Purohit M and Srivastava S K, Proc Natl Acad Sci India., 1991, 61A, 461.

4. Kraatz H-B and Metzler-Nolte N, Concepts and Models in Bioinorganic Chemistry. Weinheim, Germany: Wiley-VCH; 2006.

5. Lippard S J and Berg J M, Principles of Bioinorganic Chemistry. Mill Valley, Calif, USA: University Science Books, 1994.

6. Steed J W and Atwood J L, Supramolecular Chemistry Chichester, UK: Wiley; 2000.

7. Pedersen C J, J Am Chem Soc., 1967, 89, 7017.

8. a) Collins T J, Kostka K L, Munck E and Uffelman E S, J Am Chem Soc.,1990, 112, 5637.; b) Kimura E, Pure Appl Chem., 1989, 61, 823.

9. a) Shamsipur M, Mizani F, Mousavi M F, Alizadeh N, Alizadeh K, Eshghi H and Karami H, Anal Chim Acta, 2007, 22, 589.; b) Shamsipur M, Fakhari A R, Sharghi H, Eshghi H and Ganjali M R, Pol J Chem., 2002, 76, 1665.; c) Shamsipur M, Rouhani S, Ganjali M R, Sharghi H and Eshghi H, Sens Actuators B., 1999, 59, 30.; d) Shamsipur M, Rouhani S, Ganjali M R , Eshghi H and Sharghi H, Microchem J., 1999, 63, 202; e) Shamsipur M, Rouhani S, Sharghi H, Ganjali M R and Eshghi H, Anal Chem., 1999, 71, 4938.

10. Bubmis B D and Paciy G E, Tetrahedron Lett., 1984, 25, 1107.

11. Hiraoka M, Crown Compounds. Their Characteristics and Applications, Elsevier Scientific Publishing Company, New York, 1982.

12. Luboch E, Cygan A and Biernat J F, Tetrahedron, 1990, 46, 2461.

13. Zhiqiang Shi and Randolph P Thummel, J Org Chem., 1995, 60, 5935.

14. Yi Yuan, Ge Gao, Zong-Lin Jiang, Jin-Song You, Zhong-Yuan Zhou, De-Qi Yuan and Ru-Gang Xie, Tetrahedron, 2002, 58, 8993.

15. Baggaley K H, Heald M, Hindley R M, Morgan B, et al., J Med Chem., 1975, 18, 833.

16. Lee H M, Lu C Y, Chen C Y, Chen W L, et al., Tetrahedron, 2004, 60, 5807. 
17. Begtrup M and Larsen P, Acta Chem Scand., 1990, 44, 1050.

18. Prager R H and sopelas C, Aust J Chem., 1990, 43, 367.

19. Allin S M, Bowman W R, Elsegood M R J, McKee V, et al., Tetrahedron, 2005, 61, 2689.

20. Sonegawa M, Tokota M, Tomiyama H and Tomiyama T, Chem Pharm Bull., 2006, 54, 706.

21. Tang Y, Dong Y, Wittlin S, Charman S A, et al., Bioorg Med Chem Lett., 2007, 17, 1260.

22. Benjes P and Grimmett R, Heterocycl., 1994, 37, 735. 


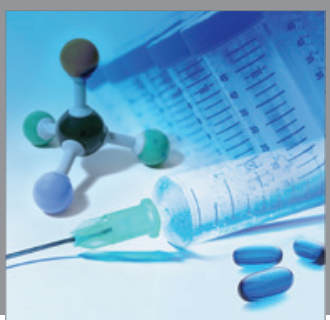

International Journal of

Medicinal Chemistry

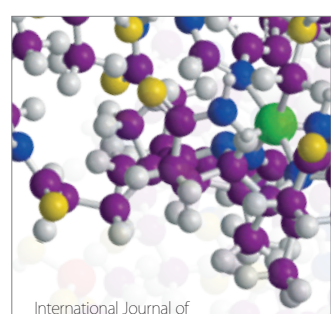

Carbohydrate Chemistry

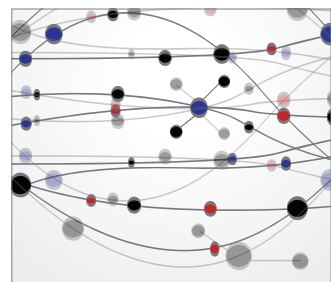

The Scientific World Journal
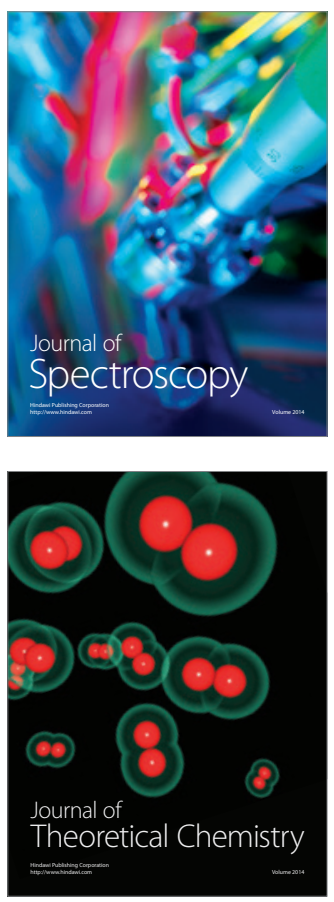
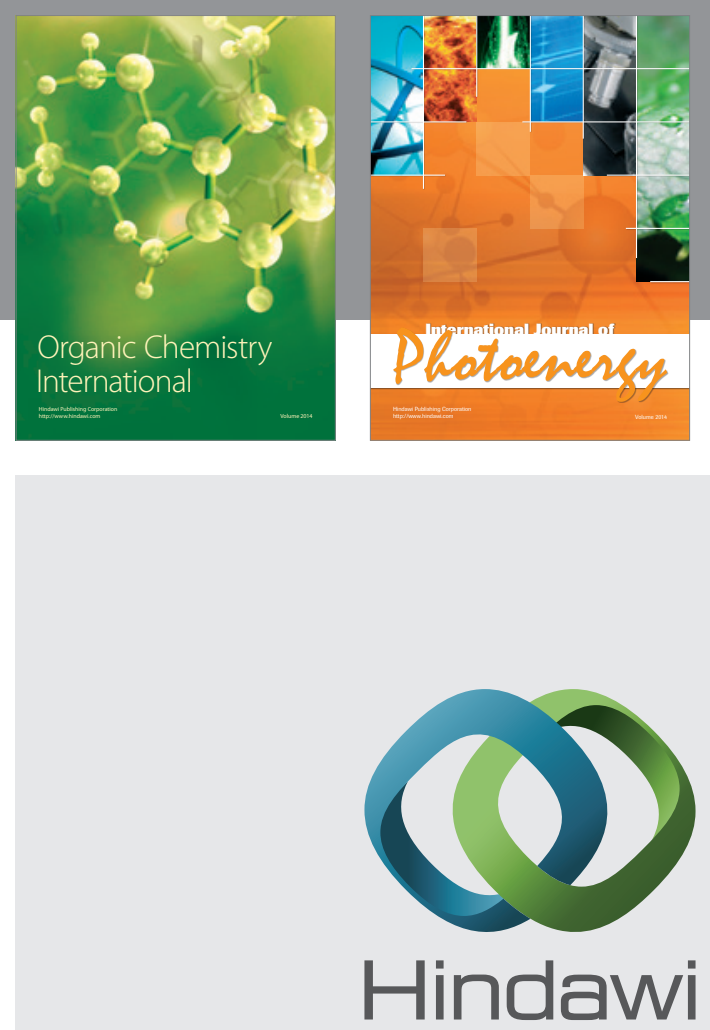

Submit your manuscripts at

http://www.hindawi.com
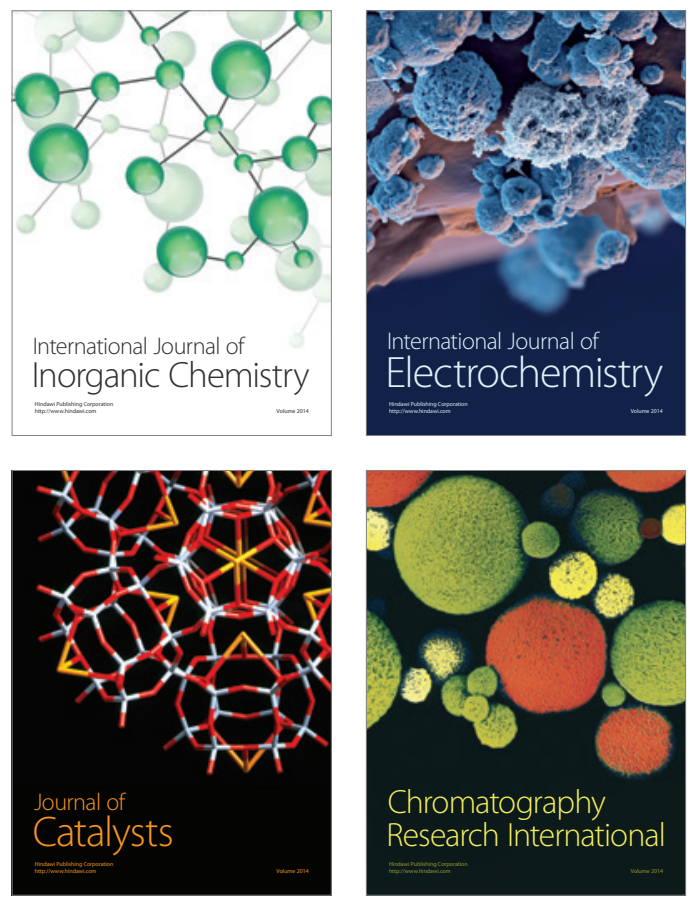
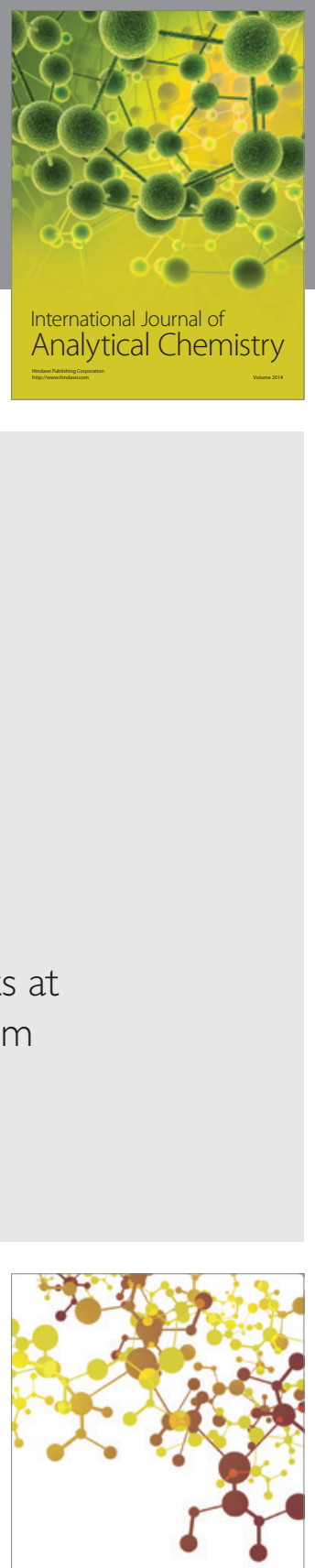

Journal of

Applied Chemistry
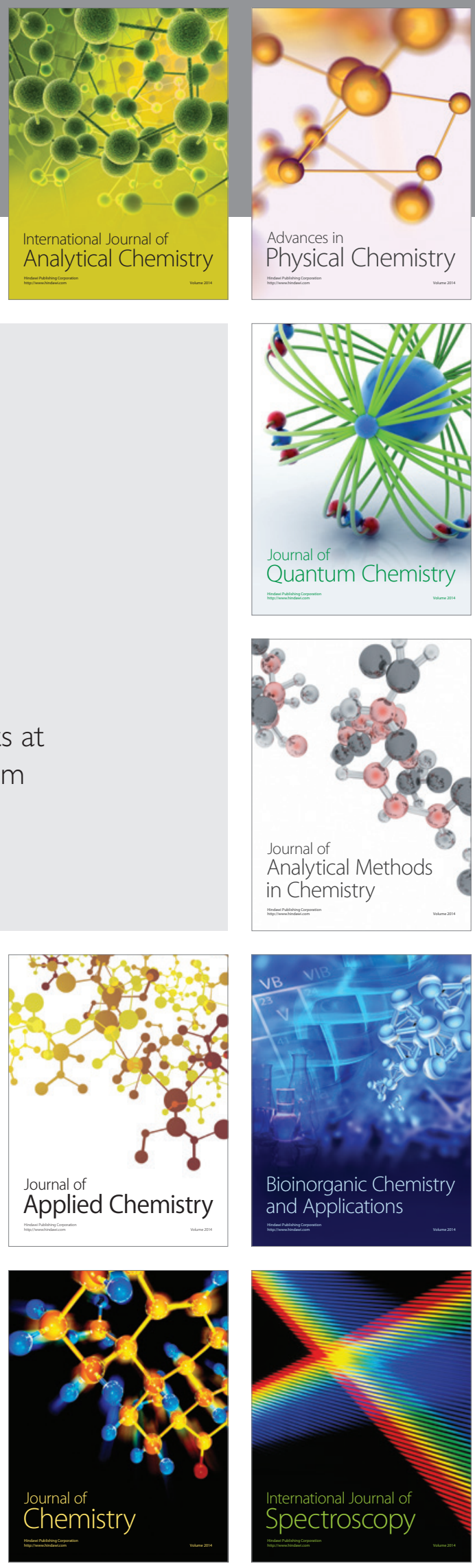